\title{
Reduzierte Dosis von TKI führt nicht zu Wirkungs- verlust bei Patienten mit aktivierender EGFR-Mutation
}

\section{A Reduced TKI Dosage is not Associated with Loss of Activity in EGFR Mutated NSCLC Patients}

Autoren

Institute
N. Reinmuth ${ }^{1,3}$, D. Heigener ${ }^{1,3}$, T. Goldmann ${ }^{1,2}$, I. Watermann ${ }^{1,2,3}$, M. Reck ${ }^{1,3}$

LungenClinic Grosshansdorf, Onkologischer Schwerpunkt

2 Forschungszentrum Borstel, Klinische und Experimentelle Pathologie

Area Research Center North, Deutsches Zentrum für Lungenforschung (DZL) eingereicht 3.6.2014 akzeptiert nach Revision 5.8.2014

\section{Bibliografie}

Dol http://dx.doi.org/ 10.1055/s-0034-1377927 Pneumologie 2014; 68: 619-623 (c) Georg Thieme Verlag KG Stuttgart · New York ISSN 0934-8387

\section{Korrespondenzadresse} PD Dr. med. Niels Reinmuth LungenClinic Onkologischer Schwerpunkt Wöhrendamm 80 22927 Großhansdorf N.Reinmuth@lungenclinic.de

\section{Zusammenfassung \\ $\nabla$}

Einleitung: Die Auswirkungen einer bei Toxizität notwendigen Dosisreduktion auf die Wirksamkeit von Tyrosinkinaseinhibitoren (TKI) bei Patienten mit aktivierender EGFR-Mutation sind unbekannt.

Methodik: Patienten mit Stadium IV NSCLC, die 2012 und danach eine TKI-Behandlung bei nachgewiesener aktivierender EGFR-Mutation erhielten, wurden retrospektiv bezüglich Dosierung und klinischem Verlauf analysiert.

Ergebnisse: Es wurden 30 Patienten mit Adenokarzinom und aktivierender EGFR-Mutation identifiziert, die vorwiegend in unserer Klinik therapiert wurden. Elf Patienten erhielten zunächst eine Chemotherapie, bevor eine TKI-Therapie eingeleitet wurde. Alle Behandlungen mit EGFR TKI führten zu einer Krankheitskontrolle (Ansprechrate 77\%) bei einem medianen progressionsfreien Überleben von 21,4 Monaten. Bei 21 Patienten wurde zum Datenerhebungszeitpunkt die TKI-Therapie aktuell fortgeführt bei einer mittleren Behandlungsdauer von 11,6 Monaten. Bei 7 Patienten wurde aufgrund Komorbiditäten $(\mathrm{n}=2)$ oder Hauttoxizität ( $\geq$ CTC Grad $3 ; n=5)$ eine Dosisreduktion von Erlotinib notwendig. Diese 7 Patienten zeigten ein ähnliches Ansprechen und einen ähnlichen Verlauf im Vergleich zu Patienten ohne Dosisreduktion und TKI-Therapie.

Schlussfolgerung: Klinisch notwendige Dosisreduktionen von EGFR TKI scheinen nicht mit einem Wirkungsverlust einherzugehen.

\section{Hintergrund}

$\nabla$

Der epidermale Wachstumsfaktor-Rezeptor (EGFR) ist in eine Vielzahl von wichtigen Signalübertragungswegen des Tumorstoffwechsels eingebunden [1]. Eine pathophysiologische Daueraktivierung der Tyrosinkinase-Aktivität durch

\section{Abstract \\ $\nabla$}

Background: Patients treated with anti-EGFR tyrosine kinase inhibitors (TKI) may receive dose reduction due to toxicity; however, the impact on treatment efficacy and patient outcome remains unknown.

Patients and Methods: Patients with stage IV NSCLC harbouring EGFR mutations and treated at our institution were retrospectively analyzed for treatment with TKI in 2012 or later.

Results: Thirty patients with EGFR mutated adenocarcinoma were identified meeting the inclusion criteria. Eleven patients received cytotoxic therapy as first line treatment yielding in a response rate of $36 \%$. All patients were treated with TKI as first or second-line therapy which resulted in disease stabilization (23\%) or response (77\%) in all patients. The median progressionfree survival was 21.4 months with 21 patients still on treatment with TKI.

For 7 patients, the dose of TKI treatment had to be reduced due to co-morbidities $(n=2)$ or skin toxicity (rash $\geq$ grade $3 ; n=5$ ). These patients demonstrated a similar response and outcome as compared to patients receiving full dose TKI treatment.

Conclusion: Patients with EGFR mutated NSCLC and the need for TKI dose reduction seem to benefit in a similar manner from TKI therapy. 
kleinzelligen Lungenkarzinom (NSCLC) identifiziert werden mit einer erhöhten Inzidenz bei Nichtrauchern und AdenokarzinomEntität, weshalb eine prätherapeutische Testung zumindest bei diesen Patientenkollektiven empfohlen wird [2].

Der Stellenwert einer Therapie mit Tyrosinkinaseinhibitoren (TKI) bei Patienten mit einer aktivierenden EGFR-Mutation wurde in mehreren, großen randomisierten Studien belegt und zeigt im Vergleich zur Chemotherapie ein überlegenes Ansprechen und verbessertes progressionsfreies Überleben (PFS) bei höherer Lebensqualität [3]. Aktuell sind für die Therapie des metastasierten NSCLCs mehrere Medikamente zugelassen, um EGFR-Tyrosinkinasen zu hemmen: die EGFR TKIs Erlotinib, Gefitinib und Afatinib, die alle ein ähnliches Nebenwirkungsprofil zeigen. Trotz einer generell guten Verträglichkeit aller TKIs erzwingen in der klinischen Praxis manchmal Nebenwirkungen wie akneiformer Hautausschlag oder Diarrhoe doch eine Dosisreduktion, wobei eine mögliche Beeinflussung auf die Therapieeffektivität unklar ist. Zudem gibt es bislang keine Studien, die die Wirksamkeit der drei zugelassenen TKIs miteinander direkt vergleichen.

\section{Patienten und Methoden}

Die Auswertung umfasste alle Patienten mit NSCLC im Stadium IV und nachgewiesener aktivierender EGFR-Mutation, bei denen vom 1.1.2012 bis zum 31.12.2013 eine Therapie mit einem antiEGFR TKI durchgeführt wurde. Es wurden nur Patienten berücksichtigt, die vorwiegend in der LungenClinic Grosshansdorf mit einem anti-EGFR TKI über eine Dauer von mindestens 8 Wochen behandelt wurden, um eine Beurteilung des Ansprechens zu ermöglichen. Bei Adenokarzinomen wurde zusätzlich der dominierende Subtyp nach der aktuellen IASLC-Klassifikation bestimmt (lepidisch, azinär, papillär, micropapillär, solide).

Es wurden sämtliche verfügbaren Daten bezüglich des Krankheitsverlaufes und der applizierten Therapien einschließlich Dosierung und Nebenwirkungen erfasst. Für Stadieneinteilung und Beurteilung des Ansprechens wurden die 7. Auflage der UICC-Kriterien sowie die Recist-Kriterien (Version 1.1) verwendet. Patienten mit Konsum von weniger als 100 Zigaretten im Leben wurden als Nieraucher klassifiziert. Alle Patienten wurden während einer Therapie mindestens alle 3 Wochen (4 Wochen für TKI) mittels Bildgebung kontrolliert. Nach Therapieende wurden regelmäßige Nachsorge-Untersuchungen durchgeführt [1]. Es wurde das am besten erreichte Ansprechen analysiert (best disease control rate, BDCR) [4]. Auftretende Toxizitäten wurden nach den Common Terminology Criteria for Adverse Events (CTCAE, Version 4.03) klassifiziert.

\section{Statistische Analyse}

Die Datenerhebung wurde am 28.2.2014 abgeschlossen. Als Gesamtüberleben wurde das Intervall zwischen Therapiebeginn und Todeszeitpunkt bestimmt. Lebende Patienten wurden zensiert. Die Todesursache wurde nach klinischen Kriterien definiert. Das progressionsfreie Überleben wurde von Therapieinitiierung bis Therapieabbruch errechnet. Die statistische Auswertung erfolgte elektronisch mit SPSS für Windows (Version 16.0.1). Univariate Analysen wurden mit Hilfe des log-rank-Regressionstests berechnet, wobei Ergebnisse mit $\mathrm{p}<0,05$ als signifikant gewertet wurden.
Tab. 1 Patientencharakteristika.

\begin{tabular}{|llcc|}
\hline & & Anzahl & Prozent \\
\hline Geschlecht & männlich & 9 & 30 \\
\hline & weiblich & 21 & 70 \\
\hline $\begin{array}{l}\text { Medianes Alter } \\
\text { Medianes Alter } \\
\text { bei Beginn TKI }\end{array}$ & & 67 Jahre & \\
\hline Raucherstatus & Nieraucher & 68,2 Jahre & \\
\hline & Ex-Raucher >6 Monate & 11 & 45,8 \\
\hline & Ex-Raucher<6 Monate & 2 & 45,8 \\
\hline & unbekannt & 6 & \\
\hline & mediane PJ & 13,5 PJ & \\
\hline Adenokarzinom, & lepidisch & 5 & 17 \\
dominanter Subtyp & azinär & 3 & 10 \\
\hline & papillär & 1 & 3 \\
\hline & solide & 9 & 30 \\
\hline & nicht bestimmbar & 12 & 40 \\
\hline & Stadium IV & 30 & 100 \\
\hline Stadium & 19 & 18 & 60 \\
\hline Exon & 21 & 12 & 40 \\
\hline
\end{tabular}

TKI, Tyrosinkinaseinhibitor.

Tab. 2 Erstlinien-Chemotherapie bei EGFR mutiertem NSCLC.

\begin{tabular}{|llll|}
\hline & & Anzahl & Prozent \\
\hline Medikation & Platin-basiert & 9 & 81,8 \\
\hline & Studie & 2 & 18,2 \\
\hline Ansprechen & PR & 4 & 36,4 \\
\hline & SD & 7 & 63,6 \\
\hline $\begin{array}{l}\text { Ansprechen Platin-basierte } \\
\text { Chemotherapie }\end{array}$ & PR & 4 & 44,4 \\
\hline & SD & 5 & 55,6 \\
\hline Medianes PFS & 5 Monate & & \\
\hline
\end{tabular}

PFS, progressionsfreies Überleben; PR, partielle Remission; SD, stabile Erkrankung.

\section{Ergebnisse \\ $\nabla$}

Es wurde bei insgesamt 41 Patienten mit NSCLC im Stadium IV zwischen 2012 und 2013 eine anti-EGFR-Therapie durchgeführt. Hiervon wurden 30 Patienten (21 weiblich; 9 männlich) identifiziert, die vorwiegend in unserer Klinik therapiert wurden $(\bullet$ Tab. 1). Alle Patienten hatten ein Adenokarzinom mit Mutationen in Exon 21 (L858 R) oder Deletionen in Exon 19 des EGFRGens. Bei Beendigung der Datenerhebung lebten noch 25 Patienten bei einer medianen Überlebenszeit von 21,4 Monaten.

Elf Patienten erhielten zunächst eine Chemotherapie (Platin-basierte Kombinationstherapie, $\mathrm{n}=9$; Therapie in klinischer Studie, $\mathrm{n}=2$ ). Die Studientherapie bestand in beiden Fällen aus der Kombination Pemetrexed und Bevacizumab (65 Plus Studie) [5]. Bei einer Gesamtansprechrate der Chemotherapie von $36 \%$ betrug das mediane progressionsfreie Überleben 5 Monate ( $\bullet$ Tab.2).

Eine anti-EGFR-Therapie wurde als Erstlinien- oder Zweitlinientherapie mit Gefitinib oder Erlotinib durchgeführt ( $\bullet$ Tab.3, - Abb. 1). Alle Behandlungen mit anti-EGFR TKI führten zu einer Krankheitskontrolle bei einer Ansprechrate von 77\% (4 CR; 19 PR) und einer mittleren Behandlungsdauer von 13,3 Monaten (Erlotinib 14,1 Monate; Gefitinib 11,7 Monate). Bei 21 Patienten wurde zum Datenerhebungszeitpunkt die TKI-Therapie fortgeführt bei einer mittleren Behandlung von 11,6 Monaten. Eine 
Tab.3 Allgemeine Wirksamkeit einer Therapie mit anti-EGFR TKI bei EGFR mutiertem NSCLC.

\begin{tabular}{|c|c|c|c|}
\hline & & Anzahl & Prozent \\
\hline \multirow{2}{*}{ Therapielinie } & 1 & 19 & 63,3 \\
\hline & 2 & 11 & 36,7 \\
\hline \multirow[t]{3}{*}{ Ansprechen } & $C R$ & 4 & 13,3 \\
\hline & PR & 19 & 63,3 \\
\hline & SD & 7 & 23,3 \\
\hline \multirow[t]{2}{*}{ TKI } & Gefitinib & 10 & 33,3 \\
\hline & Erlotinib & 20 & 66,7 \\
\hline \multirow[t]{2}{*}{ Initialdosis Erlotinib } & $150 \mathrm{mg}$ & 18 & $90 \%$ \\
\hline & $100 \mathrm{mg}$ & 2 & $10 \%$ \\
\hline \multirow[t]{2}{*}{ Reduktion Erlotinib } & ja & 5 & $25 \%$ \\
\hline & nein & 15 & $75 \%$ \\
\hline
\end{tabular}

TKI, Tyrosinkinaseinhibitor; CR, komplettes Ansprechen; PR, partielle Remission; SD, stabile Erkrankung.

Therapie mit einem TKI erst ab der zweiten Linie führte nicht zu einem schlechteren Ansprechen $(p=0,172)$ oder progressionsfreiem Überleben (Mediane 16,7 versus 22 Monate als Erstlinienversus Zweitlinientherapie; $p=0,164 ; 0$ Abb. 2).

Bei 5 Patienten wurde aufgrund von Hauttoxizität $\mathrm{CTC} \geq \operatorname{Grad} 3$ im Therapieverlauf die Erlotinib-Dosis auf $100 \mathrm{mg}$ reduziert (3 Patienten nach 1 Monat, 1 Patient nach 78 Tage, 1 Patient nach 766 Tage). Zudem erhielten 2 weitere Patienten bereits bei Therapiebeginn eine Dosis von $100 \mathrm{mg}$ Erlotinib (bei PS ECOG 2 oder Empyem). Eine Dosis-Reeskalation nach klinischer Stabilisierung wurde von keinem Patienten gewünscht. Bei Gefitinib wurde keine Dosismodifikation vorgenommen ( $\bullet$ Tab.4). Patienten mit Erlotinib-Dosisreduktion hatten kein unterschiedliches Ansprechen oder PFS im Vergleich zu Patienten mit regulärer Dosierung des EGFR TKI (Mediane 21,4 Monate versus 22 Monate; - Abb.3). Ähnliche Daten zeigten sich für behandelte Patienten mit reduzierter Erlotinib Dosis im Vergleich zu Patienten mit regulärer Erlotinib-Dosis (nicht gezeigt).

Afatinib, das zum Analysezeitpunkt noch nicht zur Therapie von TKI-naiven NSCLC-Patienten mit aktivierender EGFR-Mutation zugelassen war, wurde bei drei Patienten direkt nach Progress unter Erlotinib (2 Patienten) oder Gefitinib (1 Patient) im Rahmen eines Compassionate Use Programmes eingesetzt und führte dort jeweils zu einer erneuten Krankheitskontrolle von 4, 6, und 11 Monaten.

\section{Zusammenfassung und Diskussion}

Patienten mit NSCLC und aktivierender EGFR-Mutation sollten mit einem anti-EGFR TKI behandelt werden. Die Prävalenz in einem großen kaukasischen Kollektiv betrug abhängig vom histologischen Subtyp insgesamt 7\% [6], was unseren Daten ähnelt [7]. Trotz einer insgesamt guten Verträglichkeit kommt es bei manchen Patienten doch zu Nebenwirkungen, die eine Dosisreduktion aus klinischer Sicht notwendig machen. Die aktuelle Studie untersuchte retrospektiv in einem Lungenkrebszentrum die Auswirkungen einer klinisch indizierten Dosisreduktion auf die Wirksamkeit und das Überleben von TKI-behandelten Patienten.

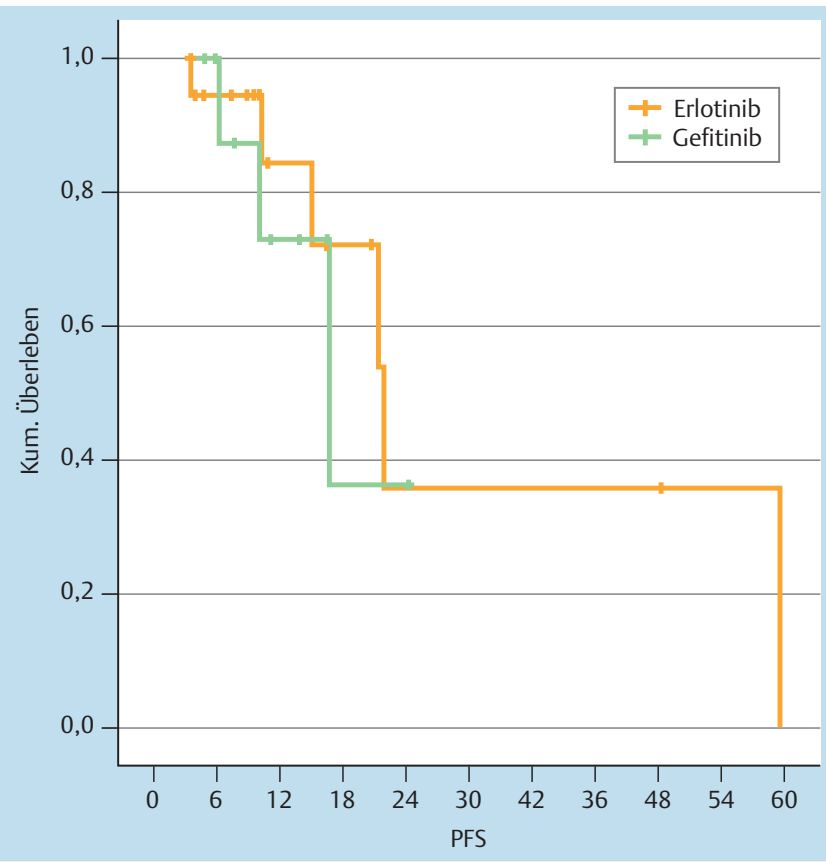

Abb. 1 Progressionsfreies Überleben unter einer Therapie mit anti-EGFR TKI. Patienten mit EGFR mutiertem NSCLC im Stadium IV wurden mit Gefitinib oder Erlotinib als Erst-oder Zweitlinientherapie behandelt. Es wurde kein signifikanter Unterschied zwischen Erlotinib und Gefitinib bezüglich progressionsfreiem Überleben beobachtet $(p=0,724)$.

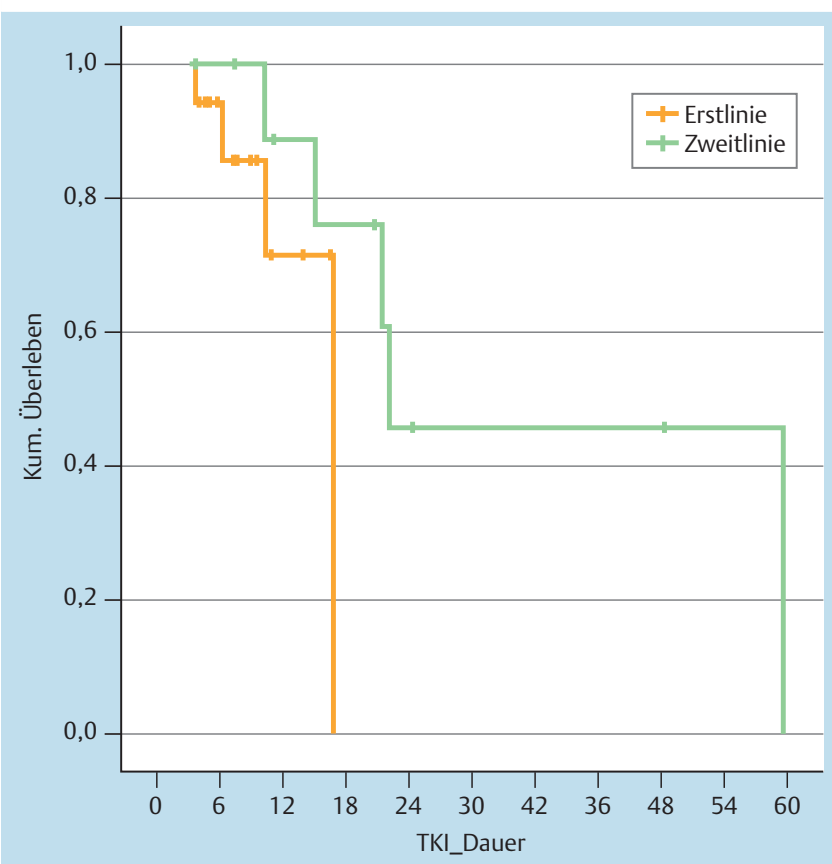

Abb.2 Vergleich einer anti-EGFR-TKI-Behandlung als Erstlinien- versus Zweitlinientherapie. Das progressionsfreie Überleben war bei einer Behandlung mit einem anti-EGFR TKI als Zweitlinientherapie nicht signifikant verschieden von einer anti-EGFR-Erstlinientherapie $(p=0,164)$. 
Tab.4 Wirksamkeit einer Therapie mit anti-EGFR TKI in unterschiedlicher Dosis.

\begin{tabular}{|llcc|}
\hline & Bestes Ansprechen & Anzahl & Prozent \\
\hline Erlotinib $150 \mathrm{mg} /$ Tag & CR & 1 & 7,7 \\
\hline & PR & 10 & 76,9 \\
\hline & SD & 2 & 15,4 \\
\hline Med. PFS & 22 Monate & & \\
\hline Erlotinib $100 \mathrm{mg} /$ Tag & CR & 1 & 14,3 \\
\hline & PR & 4 & 57,1 \\
\hline & SD & 2 & 28,6 \\
\hline Med. PFS & 20,4 Monate & & \\
\hline Gefitinib & CR & 2 & 20 \\
\hline & PR & 5 & 50 \\
\hline & SD & 3 & 30 \\
\hline Med. PFS & 16,7 Monate & & \\
\hline
\end{tabular}

PFS, progressionsfreies Überleben; CR, komplettes Ansprechen; PR, partielle Remission; $\mathrm{SD}$, stabile Erkrankung.

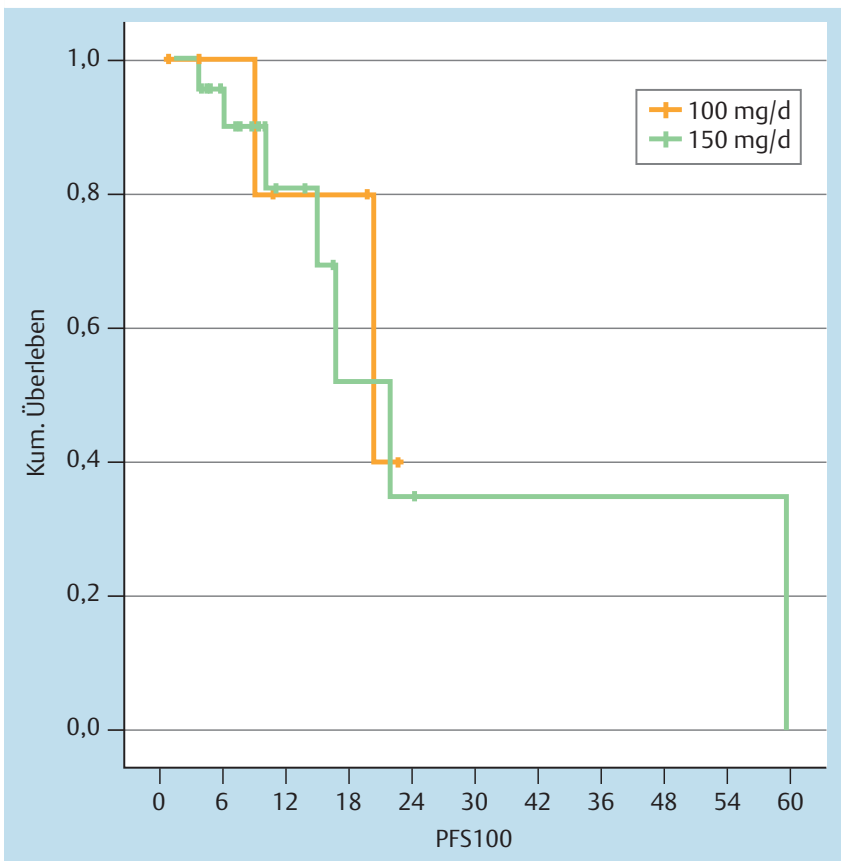

Abb. 3 Progressionsfreies Überleben bei EGFR TKI in unterschiedlicher Dosierung. Bei 7 Patienten musste bei klinischer Indikation eine Therapie mit Erlotinib in reduzierter Dosierung durchgeführt werden. Dabei war das progressionsfreie Überleben für diese Patienten nicht signifikant verschieden von Patienten, die Gefitinib oder Erlotinib in regulärer Dosis erhielten $(p=0,71)$.

Eine Dosisreduktion musste bei 35\% der mit Erlotinib-behandelten Patienten vorgenommen werden. Die Reduktion der Dosis wurde ausschließlich aufgrund klinischer Kriterien wie vermehrter und den Patienten belastender Hautausschlag oder reduzierter Allgemeinzustand vorgenommen. Aufgrund des retrospektiven Charakters der Untersuchung sind Rückschlüsse auf detaillierte Häufigkeitsangaben von Nebenwirkungen bei den einzelnen Präparaten allerdings nicht möglich [8]. Interessanterweise ging eine Dosisreduktion nicht signifikant mit einem Wirkungs- verlust bezüglich des Ansprechens oder des progressionsfreien Überlebens einher. Auch wenn diese Daten nur in einem zahlenmäßig begrenzten Kollektiv erhoben wurden, so ist die vorliegende Analyse die erste Auswertung von kaukasischen Patienten mit anti-EGFR-Therapie.

Bisher wurden nur sehr spärliche Daten zu möglichen Auswirkungen auf die Effektivität von TKIs bei Dosisreduktionen berichtet. In einer retrospektiven Analyse von 55 japanischen Patienten mit unterschiedlichem Mutationsstatus, die mit Erlotinib behandelt wurden und einen Hautausschlag mit einem Schweregrad höher als 2 entwickelten, zeigten 24 Patienten mit nachfolgender Dosisreduktion ein längeres medianes PFS als Patienten ohne Dosisreduktion (341 versus 70 Tage, $\mathrm{p}<0,001$ ) sowie ein besseres Gesamtüberleben (566 versus 202 Tage; $p<0,001$ ) [9]. Allerdings waren in dieser Studie in der Patientengruppe mit Erlotinib-Dosisreduktion signifikant mehr Patienten mit EGFR-Mutationen ( $63 \%$ vs $32 \%, p=0,032$ ) enthalten, weswegen die Interpretation dieser Daten sehr schwierig wird.

Da die Dosierung von Gefitinib und Erlotinib bezüglich der maximal tolerierbaren Dosis (MTD) sehr unterschiedlich ist [10], wurden immer wieder Spekulationen über unterschiedliche Wirksamkeiten angestellt. Ein Vergleich zwischen Gefitinib- und Erlotinib-Therapie ergab in unserer Untersuchung jedoch keinen signifikanten Unterschied im progressionsfreien Überleben. Auch hier wurden bisher kaum Daten berichtet. In einer retrospektiven Analyse von 154 japanischen Patienten mit NSCLC fand sich kein signifikanter Unterschied im Ansprechen zwischen einer Behandlung mit Gefitinib (85 Patienten) oder Erlotinib (69 Patienten) [11]. Allerdings wurde nur bei 59 Patienten in diesem Kollektiv überhaupt eine EGFR-Mutation nachgewiesen (Wildtyp bei 30; nicht untersucht bei 65). Gefitinib wurde signifikant häufiger bei Patienten mit EGFR-Mutationen eingesetzt ( $52 \%$ versus $23 \%, p=0,03$ ), was einen Trend zu verbessertem Ansprechen für Gefitinib behandelte Patienten im Vergleich zu Erlotinib behandelten Patienten ( $52 \%$ versus $36 \%, p=0,11$ ) erklärt. Eine aktuelle Phase-IIb-Studie untersucht prospektiv die Wirkung einer Erstlinientherapie mit Afatinib versus Gefitinib bei Patienten mit Adenokarzinomen und aktivierender EGFR-Mutation (Lux-Lung 7, NCT01466660).

Schließlich untersuchten wir die Effektivität einer ErstlinienChemotherapie bei Patienten mit EGFR-Mutation. Die Ansprechrate auf eine Platin-haltige Kombinationstherapie lag bei 44\%. Ein ähnliches Ergebnis wurde mit 47,3\% bei 129 asiatischen Patienten mit EGFR mutiertem Adenokarzinom nach einer Therapie mit Carboplatin/Paclitaxel beschrieben [12]

Diese Daten deuten ein leicht besseres Ansprechen auf eine zytotoxische Therapie bei EGFR mutierten NSCLC-Patienten an im Vergleich zu unselektierten Patienten, bei denen Ansprechraten um $30 \%$ berichtet wurden [13]. In einer retrospektiven Analyse wurden bei nicht-squamösen Patienten mit Platin-haltiger Kombinationschemotherapie sogar ähnliche Ansprechraten (41\%) beobachtet [4].

Insgesamt scheinen Dosisreduktionen von EGFR TKI, die aufgrund von Toxizität oder reduziertem Allgemeinzustand notwendig werden, nicht mit einem Wirkungsverlust einherzugehen. Randomisierte Daten sowie Daten aus größeren Kollektiven fehlen hierzu. Daher sollte, wenn klinisch möglich, weiterhin eine Therapie mit EGFR TKIs in normaler Dosis durchgeführt werden. 


\section{Interessenkonflikt}

N. Reinmuth erhielt Honoraria für Vorträge von Hoffmann-La Roche, Lilly, Novartis, Boehringer-Ingelheim, Otsuka und BristolMyers Squibb und für Beratungen von Hoffmann-La Roche, Lilly, Amgen, Novartis und Bristol-Myers Squibb.

D. Heigener erhielt Honoraria für Vorträge von Boehringer-Ingelheim, Lilly, Hoffmann-La Roche, Novartis Bristol-Myers Squibb und Pfizer und für Beratungen von Boehringer-Ingelheim, Hoffmann-La Roche, Lilly, Pfizer.

M. Reck erhielt Honoraria für Vorträge von Hoffmann-La Roche, Lilly, BMS, Novartis, Boehringer-Ingelheim, Pfizer, AstraZeneca und für Beratungen von Hoffmann-La Roche, Lilly, BMS, Novartis, Boehringer-Ingelheim, Pfizer, AstraZeneca.

T. Goldmann und I. Watermann geben an, dass kein Interessenkonflikt besteht.

\section{Literatur}

1 Goeckenjan G, Sitter H, Thomas M et al. Prevention, diagnosis, therapy, and follow-up of lung cancer: interdisciplinary guideline of the German Respiratory Society and the German Cancer Society. Pneumologie 2010; 65: 39-59

2 Pao W, Girard N. New driver mutations in non-small-cell lung cancer. Lancet Oncol 2011; 12: 175-180

3 Lee CK, Brown C, Gralla RJ et al. Impact of EGFR inhibitor in non-small cell lung cancer on progression-free and overall survival: a meta-analysis. J Natl Cancer Inst 2013; 105: 595-605

4 Reinmuth $N$, Payer $N$, Muley $T$ et al. Treatment and outcome of patients with metastatic NSCLC: a retrospective institution analysis of 493 patients. Respir Res 2013; 14: 139
5 Schuette $W$, Nagel S, Schneider CP et al. 65 plus: A randomized phase III trial of pemetrexed and bevacizumab versus pemetrexed, bevacizumab, and carboplatin as first-line treatment for elderly patients with advanced nonsquamous, non-small cell lung cancer (NSCLC). J Clin Oncol 2013; 31: suppl: abstr 8013

6 Rosell R, Carcereny E, Gervais $R$ et al. Erlotinib versus standard chemotherapy as first-line treatment for European patients with advanced EGFR mutation-positive non-small-cell lung cancer (EURTAC): a multicentre, open-label, randomised phase 3 trial. Lancet Oncol 2012; 13: 239-246

7 Kanappilly A, Heigener DF, Reck $M$ et al. Systematischer molekularpathologischer Nachweis von EGFR, KRAS und EML4-ALK an zytologischem Material. Pneumologie 2014; 68: P73

8 Sebastian M, Schmittel A, Reck M. First-line treatment of EGFR-mutated nonsmall cell lung cancer: critical review on study methodology. Eur Respir Rev 2014; 23: $92-105$

9 Takashima N, Kimura T, Watanabe $N$ et al. Prognosis in patients with non-small cell lung cancer who received erlotinib treatment and subsequent dose reduction due to skin rash. Onkologie 2012; 35: 747 752

10 Hughes AN, O'Brien ME, Petty WJ et al. Overcoming CYP1A1/1A2 mediated induction of metabolism by escalating erlotinib dose in current smokers. J Clin Oncol 2009; 27: 1220-1226

11 Togashi Y, Masago K, Fujita S et al. Differences in adverse events between $250 \mathrm{mg}$ daily gefitinib and $150 \mathrm{mg}$ daily erlotinib in Japanese patients with non-small cell lung cancer. Lung Cancer 2011; 74: 98 102

12 Mok TS, Wu YL, Thongprasert S et al. Gefitinib or carboplatin-paclitaxel in pulmonary adenocarcinoma. N Engl J Med 2009; 361: 947-957

13 Li M, Zhang $Q$ Fu $P$ et al. Pemetrexed plus platinum as the first-line treatment option for advanced non-small cell lung cancer: a metaanalysis of randomized controlled trials. PLoS One 2012; 7: e37229 